International Journal of Pattern Recognition

and Artificial Intelligence

Vol. 33, No. 6 (2019) 1992002 (1 page)

(c) World Scientific Publishing Company

DOI: 10.1142/S0218001419920022

\title{
Erratum \\ Bayes Performance of Batch Data Mining Based on Functional Dependencies
}

[International Journal of Pattern Recognition and Artificial Intelligence, Vol. 33, No. 3 (2019) 1959011 (15 pages), DOI: 10.1142/S0218001419590110]

Haixu $\mathrm{Xi}^{*}$, Feiyue $\mathrm{Ye}^{\dagger}$, Sheng He, Yijun Liu and Hongfen Jiang

School of Computer Engineering

Jiangsu University of Technology

Changzhou, P. R. China

*xihaixu@jsut.edu.cn

†yfy@jsut.edu.cn

Published 10 April 2019

We would like to make the following corrections to this article.

(1) The corresponding author for this paper is Feiyue Ye, not Haixu Xi.

(2) The acknowledgments section should read as follows:

This work was supported by National Natural Science Fund of China (61472166) and the 2018 Humanities and Social Science Research Fund of the Ministry of Education in China (18YJC840045). 